Authors:

Arezoo Eshraghi, $\mathrm{PhD}$

Noor Azuan Abu Osman, PhD

Mohammad Taghi Karimi, PhD

Hossien Gholizadeh, MEngSc

Sadeeq Ali, MEngSc

Wan Abu Bakar Wan Abas, PhD

\title{
Affiliations:
}

From the Department of Biomedical

Engineering, Faculty of Engineering,

University of Malaya, Kuala Lumpur,

Malaysia (AE, NAAO, HG, SA, WABWA);

and the Department of Orthotics and

Prosthetics, Faculty of Rehabilitation

Sciences, Isfahan University of Medical

Sciences, Isfahan, Iran (MTK).

Correspondence:

All correspondence and requests for

reprints should be addressed to: Arezoo

Eshraghi, PhD, Department of

Biomedical Engineering, Faculty of

Engineering, University of Malaya,

Lembah Pantai, 50603 Kuala Lumpur,

Malaysia.

0894-9115/12/9112-1028/0

American Journal of Physical

Medicine \& Rehabilitation

DOI: 10.1097/PHM.0b013e318269d82a

\section{Quantitative and Qualitative Comparison of a New Prosthetic Suspension System with Two Existing Suspension Systems for Lower Limb Amputees}


ranstibial prosthetic designs incorporate suspension

systems consisting of liners and coupling

components. Manufacturers continuously seek improvement

in prosthetic components.1,2 The contours

and buildups on the polyethylene foam liner

(Pelite) worn inside the prosthetic hard socket help

retain the prosthesis. A belt or strap also sometimes

provides an extra means of security. Suspension

sleeves, pulled over the prosthesis to give extra suspension,

were introduced as an added feature, and

later, silicone liners were invented to improve suspension

by establishing a firm bond between the

residual limb and the liner.3,4 Internal pin lock systems

and, recently, single or multiple hypobaric seals

around the liners were developed as alternatives to

external accessories. Improved suspension has been

reported in objective and subjective studies as an

advantage of silicone liners. 4 Silicone liners are less

bulky than other types of suspension. Enhanced

suspension and cosmesis have produced higher

satisfaction rates among transtibial amputees.5,6

Satisfaction is said to be correlated with low

piston motion, decreased unwanted sounds during

functional tasks, and ease of don and doff.7Y9 A suspension

system should not only retain the prosthesis

to the residual limb but also provide comfort, enhanced

function, and ease of don and doff. The ease

and simplicity of donning and doffing are of critical

importance among prosthetic users.10,11 The users

have reported difficulty in the proper alignment

of pins in the pin lock systems. These systems may

also cause a phenomenon called Bmilking[ caused

by tissue stretch at the pin site, particularly during

the swing phase of gait.12,13 This milking might be

the cause of pain and discomfort at the distal end

of the residual limb, particularly during swing. 
Researchers have investigated the pros and cons of different transtibial suspension systems both objectively and subjectively. The studies have targeted different determinants of successful prosthetic provision; lack of pistoning has been one of the main variables that indicate proper socket fit.14 Some research studies have shown preferences for the pin lock and suction systems with total-surfaceY bearing sockets over the polyethylene foam liners used with patellar tendonYbearing sockets,4,7,15,16 which exert high pressures on the residual limb.

Pistoning is defined as the vertical displacement mainly occurring within the prosthetic socket either between the residual limb and the liner or between the liner and socket wall.17 Improper suspension might result in residual limb skin problems, gait deviations, and discomfort.8,18 Several methods have been used for measuring the pistoning inside the prosthetic socket.16 This has been mostly conducted by radiography, 8,18 ultrasound, 19 and computerized tomography.20 A recent method used a photographic technique for evaluation of piston motion between the liner and the socket.21,22 Finally, the use of motion analysis systems by reflective markers was recently introduced to measure pistoning. 7 The very same method was adopted in this study to evaluate the effect of the newly designed suspension system on pistoning.7 Pistoning measurement has been mostly performed through gait simulation because either evaluation during the real gait had been detrimental to the amputee or some technical limitations hindered the measurement during the real gait. 1

Qualitative surveys in the field of prosthetics have frequently used the Prosthesis Evaluation Questionnaire (PEQ) to investigate the effects of prostheses on the quality-of-life among individuals with amputation. Good reliability and validity have 
been reported for the PEQ.23 The PEQ research on

prosthesis satisfaction has revealed that donning

and doffing might play important roles in amputees'

satisfaction.24

Although silicone suspension systems such

as the pin lock and the hypobaric seal-in liners are

said to provide enhanced suspension for lower limb

prostheses, 4 some disadvantages such as increased

pain at the residual limb and difficulty of donning

and doffing are also attributed to them.7 To overcome

some of the disadvantages of the pin lock and

suction suspension systems, the authors of the current

study invented, produced, and evaluated a new

prosthetic suspension system compared with the

pin lock and suction systems. The purposes of this

study were to compare the new suspension system

with the two existing methods of suspension in the

pistoning motion between the prosthetic liner and

the socket and to compare satisfaction and perceived

problems of transtibial amputees. The authors hypothesized

that the new suspension system will cause

less pistoning compared with the pin lock system,

whereas the resultant pistoning will be higher than

that of the suction suspension system. The authors'

other hypothesis was that there will be a significant

increase in satisfaction rates with the new suspension

system than with the other two systems.

\section{METHODS}

\section{Participants}

Ten individuals with transtibial amputation

were selected as a convenience sample to participate in this prospective study. The inclusion criteria

were unilateral transtibial amputation, activity

levels of K2YK3 according to the American Academy

of Orthotists \& Prosthetists, 25 residual limbs

free of wound and pain, no upper limb disability,

experience with silicone liners, no volume fluctuation 
in the residual limb, and the ability to ambulate independently. The stump length, measured from the inferior edge of the patella to the distal end of the stump, had to be no less than $13 \mathrm{~cm}$. All participants used transtibial prostheses with the pin lock suspension system before the initiation of this study. Table 1 lists the individual characteristics of all subjects. The University of Malaya Ethics Committee approved this research study. The subjects were required to sign a consent form to enter this study, and the researchers considered each subject as his own control.

Three prostheses were fabricated for each subject by a single registered prosthetist to ensure uniform design, alignment, and fit. Three suspension systems were selected, including the new lower limb suspension design (Fig. 1). The other two systems were (1) the shuttle lock and pin (Dermo Liner with Icelock-clutch 4 H214 L 214000) and (2) the suction suspension (Seal-In X5 Liner with Icelock Expulsion Valve 551). Other prosthetic components were common among the three prostheses (Flex-Foot Talux and Tube adaptor).

Transparent thermoplastic material ensured that the sockets were total-surface bearing7 and had visible walls, through which the researchers could detect the internal features. The processes of checkout, gait evaluation, and gait training were performed in the Brace \& Limb Laboratory, University of Malaya. Furthermore, the PEQ required at least 1 mo of prosthetic use for each prosthetic type to allow for adaptation to the new prostheses.

\section{Full text is available at :}

http://graphics.tx.ovid.com/ovftpdfs/FPDDNCLBPCEOKH00/fs047/ovft/live/gv024/00002060/00002060- 